\title{
Routine serum biomarkers, but not Dual Energy X-ray Absorptiometry, correlate with cortical bone mineral density in children and young adults with CKD
}

Page | 1
A.D. Lalayiannis ${ }^{1}$, N.J. Crabtree ${ }^{2}$, Ferro C.J. ${ }^{3}$, V. Askiti ${ }^{4}$, A. Mitsioni ${ }^{4}$, L. Biassoni ${ }^{1}$, A. Kaur ${ }^{5}$, M.D. Sinha ${ }^{6}$, D.C. Wheeler ${ }^{7}$, N.D. Duncan ${ }^{8}$, J. Popoola ${ }^{9}$, D.V. Milford ${ }^{2}$, J. Long ${ }^{10}$, M.B. Leonard $^{10}$, M. Fewtrell ${ }^{1}$, R. Shroff ${ }^{1}$

\footnotetext{
${ }^{1}$ Great Ormond St Hospital for Children NHS Foundation Trust, and University College London Institute of Child Health, London - UK

${ }^{2}$ Birmingham Women's and Children's NHS Foundation Trust, Birmingham - UK

${ }^{3}$ University Hospitals Birmingham NHS Foundation Trust, Birmingham, UK

4"P. \& A. Kyriakou" Childrens' Hospital, Athens - Greece

${ }^{5}$ Manchester University NHS Foundation Trust, Manchester - UK

'Evelina Children's Hospital, Guy's \& St Thomas' NHS Foundation Trust, London, UK

${ }^{7}$ Department of Renal Medicine, University College London, London, UK

${ }^{8}$ Imperial College Renal and Transplant Centre, Hammersmith Hospital, London, UK.

${ }^{9}$ Department of Nephrology and Transplantation, St. George's University Hospital NHS Foundation Trust, London, UK.

${ }^{10}$ Stanford University, Palo Alto, CA, USA.
} 


\section{Abstract}

\section{Background}

Biomarkers and Dual-energy X-ray Absorptiometry (DXA) are thought to be poor predictors

Page | 2 of bone mineral density (BMD). KDIGO guidelines suggest using DXA if it will affect patient management, but this has not been studied in children or young adults in whom bone mineral accretion continues to 30 years of age. We studied the clinical utility of DXA and serum biomarkers against tibial cortical BMD measured by peripheral Quantitative CT (pQCT), expressed as Z-score (CortBMD), which predicts fracture risk.

\section{Methods}

Cross-sectional multicentre study in 26 patients with CKD 4-5 and 77 on dialysis.

\section{Results}

Significant bone pain that hindered activities of daily living was present in $58 \%$, and $10 \%$ had at least one low-trauma fracture. CortBMD and cortical mineral content Z-scores were lower in dialysis compared to CKD patients ( $p=0.004$ and $p=0.02$ ). DXA BMD hip and lumbar spine Zscores did not correlate with CortBMD or biomarkers. CortBMD was negatively associated with parathyroid hormone $(\mathrm{PTH} ; \mathrm{r}=-0.44, \mathrm{p}<0.0001)$ and alkaline phosphatase $(\mathrm{ALP} ; \mathrm{r}=-0.22$, $p=0.03)$ and positively with calcium $(r=0.33, p=0.001)$. At PTH $<3 x U L N$ (Upper Limit of Normal), none of the patients had a CortBMD below -2SD (OR 95\%Cl 7.331 to infinity).

On multivariable linear regression PTH $(\beta-0.43, p<0.0001), \operatorname{ALP}(\beta-0.36, p<0.0001)$ and calcium ( $\beta$ 0.21, $p=0.005$ ) together predicted $57 \%$ of variability in CortBMD. DXA measures did not improve this model.

\section{Conclusions}

Taken together, routinely used biomarkers, PTH, ALP and calcium, but not DXA, are moderate predictors of cortical BMD. DXA is not clinically useful and should not be routinely performed in children and young adults with CKD4-5D. 
Key words: Chronic kidney disease (CKD), Mineral Bone Disorder (MBD), peripheral

Quantitative Computed Tomography (pQCT), Dual Energy Xray Absorptiometry (DXA), bone mineralization

Page | 3

What is already known about this subject:

- The 2017 KDIGO CKD-MBD guidelines suggest using DXA imaging if it will affect patient management, but no specific recommendation is made for children and young adults.

- Dual Energy X-ray Absorptiometry has been shown to have a role in fracture prediction in the older CKD population.

- Peripheral Quantitative Computed Tomography accurately distinguishes between cortical and trabecular bone, and has shown that in children with CKD2-5D, a lower cortical bone mineral density is associated with an increased fracture risk.

What this study adds:

- CKD-MBD still leads to significant morbidity in the young CKD population (5 to 30 years old) including a high pain prevalence and atraumatic fractures.

- Routinely used serum biomarkers (calcium, parathyroid hormone, alkaline phosphatase) are only moderate independent predictors of cortical bone mineral density, and DXA imaging is not a useful adjunct.

- PTH levels under 3 times the upper limit of normal are associated with normal cortical bone mineral density.

What impact this may have on practice or policy:

- DXA imaging does not correlate with cortical bone mineral density and should not be routinely recommended as an adjunct in assessing bone health in the context of CKDMBD in the young CKD population.

- The recommended PTH target range of 2 to 9 times the upper limit of normal may be too wide; a lower target range for the young CKD population may be more appropriate

- A prospective study is required in the pediatric CKD population to assess the ability of DXA to predict fractures. 


\section{Introduction}

Mineral bone disorder (MBD) is commonly seen in children and adults with chronic kidney disease (CKD). The biochemical abnormalities [of calcium (Ca), phosphate (P),

Page I 4 parathyroid hormone (PTH) and 1,25-dihydroxyvitamin D], bone abnormalities (short stature, bone pain, deformities and increased risk of fractures) and extra-skeletal calcification $[1,2]$ together form the spectrum of CKD-MBD. This process starts early in CKD, with rising concentrations of bone derived phosphaturic hormone, Fibroblast Growth Factor 23 (FGF23), and progresses as renal function declines [3-5]. Mineral dysregulation leads to bone demineralization that, if untreated, leads to deformities and an increased fracture risk even in young CKD patients [6-8].

Assessing bone health is a key element in the management of CKD. This relies on monitoring the trend in key serum biomarkers such as PTH, Ca, P and alkaline phosphatase (ALP). The 2017 KDIGO (Kidney Disease Improving Global Outcomes) guidelines suggest that in adults with CKD3-5D, dual energy $x$-ray absorptiometry (DXA) may be used if results are likely to impact patient management [9]. This recommendation relies on evidence gathered from older people with CKD [8, 10-12]. However, in children and young adults calcium accrual in the skeleton continues until the third decade of life when peak bone mass (PBM) is achieved [13]. It has been shown that if bone mineral accretion is impaired by CKD or its treatment, this younger population is at a high risk of bone demineralization and associated fracture risk. In a longitudinal follow up study of 89 patients (aged 5-21 years), a low serum Ca level was associated with a reduction in tibial cortical bone mineral density (BMD) on peripheral quantitative computed tomography ( $\mathrm{PQCT}$ ), and $1 \mathrm{SD}$ decrease in BMD Z-score was associated with a 2-fold increase in fracture risk [14].

A detailed assessment of bone turnover and mineralization can only be achieved through bone biopsy [15]. This gold-standard method however is invasive and seldom performed in routine clinical practice. In lieu of biopsies, serum biomarkers and radiological imaging are used as surrogate markers in assessing BMD, but have some limitations that need to be taken into account. Biomarkers may not reflect the true state of bone turnover or mineralization $[16,17]$, and no biomarker individually or in combination can reliably identify mineralization or turnover defects [18]. DXA produces a two dimensional image of a three dimensional bone structure, so that cortical and trabecular bones are superimposed. 
DXA measurements are affected by bone and body size, and thus underestimates volumetric BMD $\left(\mathrm{g} / \mathrm{cm}^{3}\right)$ in children with short stature [19]. This makes its use challenging in the context of young people with CKD, who do not achieve their height potential [20].

Page 5 The imaging technique pQCT allows for measurement of volumetric BMD $\left(\mathrm{g} / \mathrm{cm}^{3}\right)$, and clearly distinguishes trabecular and cortical bone but is currently in use as a research tool only [21]. In CKD, it has been used to show progressive demineralization with CKD stage [22]. In a longitudinal follow up study decreasing cortical BMD was associated with a higher fracture risk in children with CKD2-5D [14].

This study is part of larger, prospective, longitudinal, multicentre study examining bone and cardiovascular health in children and young adults with CKD. The baseline crosssectional data relating to bone health are presented in this manuscript. Our hypothesis was that hip or lumbar spine DXA areal BMD (aBMD) was a significant predictor of volumetric BMD as measured by tibial cortical pQCT expressed as Z-score (referred to as CortBMD throughout) as determined by PQCT.

\section{Methods}

\section{Study Participants}

This cross-sectional study included children and young adults with CKD and healthy volunteers recruited from 5 pediatric hospitals and 4 adult renal units. Our inclusion criteria were: age from 5 to 30 years and CKD stages 4-5 (estimated glomerular filtration rate (eGFR; by Schwartz formula [23]) $<30 \mathrm{ml} / \mathrm{min} / 1.73 \mathrm{~m}^{2}$ ) or on dialysis. This age group was selected because children under 5 years are too small for the PQCT scanner and no reference data is available. Young adults up to 30 years were included because bone mineral accretion is shown to continue up to the third decade. We excluded any patient with malignancy, genetic or metabolic bone disease and those who would not have tolerated the scanning procedures.

A total of 145 patients were identified and 135 agreed to participate. 12 were excluded after consenting due to unwillingness to undergo some or all investigations. Onehundred and twenty three children and young adults with CKD were included. Twenty were excluded prior to analysis due to poor quality scans (see supplemental material). 
A detailed medical history, with particular relevance to bone health, was taken for all participants using a set proforma (included in supplemental material). Fracture history was self-reported and details checked in the medical records. Participants were asked to record the severity of bone pain on a numeric rating scale (where 0 indicates no pain, 5 is pain affecting activities of daily living and 10 is disabling pain causing immobility) [24].

\section{Investigations Performed}

\section{Serum Biomarkers}

Routine serum biomarkers were measured on non-fasting blood samples collected at the study visit or prior to a mid-week hemodialysis session. These included serum ionized calcium (iCa), total Ca, P, magnesium (Mg), bicarbonate, intact PTH (iPTH), 25hydroxyvitamin D [25(OH)D], and ALP. Due to different iPTH assays used at different centres, results have been expressed in multiples of upper limits of normal (ULN) (See supplemental material). As these biomarkers are measured as a part of routine clinical care, they were analysed in the patients' respective hospitals.

\section{Dual Energy X-ray Absorptiometry}

All DXA scans were undertaken by trained radiographers at the respective research centres according to the manufacturer's protocol. The lumbar spine (LS) and hips imaging were obtained according to the International Society for Clinical Densitometry (ISCD) guidelines using General Electric scanners (iDXA or Lunar) [25]. Z-scores produced by either machine have been shown to be comparable [26].

To correct the LS aBMD measurements obtained in $\mathrm{g} / \mathrm{cm}^{2}$ for height, they were converted to bone mineral apparent density (BMAD) Z-scores for participants under 20 years old. This provided age-, sex-, height- and race-specific Z-scores [26], and avoids overestimating BMD in shorter individuals, or underestimating it in taller individuals. BMAD scoring is used routinely in the UK to correct DXA measurements for poor growth or height attainment in childhood and adolescence. BMAD Z-scores for young adult LS DXA aBMD measurements were calculated assuming a maximum age of 20 years, for the purposes of 
this analysis, and referred to as DXA Z-scores throughout this document. There was no significant difference between reported aBMD Z-scores and BMAD Z-scores for the young adults.

Page | 7

\section{Peripheral Quantitative Computed Tomography}

A scan of the non-dominant tibia was obtained by pQCT as per manufacturer's instructions and ISCD guidelines using measurement points recommended by Leonard et al $[22,25,27]$. A detailed protocol is described in the supplementary material. The $3 \%$ metaphyseal and 38\% diaphyseal sites were used for image acquisition of trabecular and cortical bone respectively. The PQCT measures obtained and expressed as Z-scores were the cortical volumetric BMD $\left(\mathrm{mg} / \mathrm{cm}^{3}\right)$, trabecular volumetric BMD $\left(\mathrm{mg} / \mathrm{cm}^{3}\right)$, periosteal circumference $(\mathrm{mm})$, endosteal circumference $(\mathrm{mm})$, cortical cross-sectional area $\left(\mathrm{mm}^{2}\right)$ and cross-sectional moment of inertia (CSMI). The CSMI is a mathematical calculation that tests the efficiency of a cross-sectional shape to resist bending caused by loading stress. All results were expressed in age-, sex-, race- and height adjusted $Z$ scores derived from a reference dataset of 665 healthy children and young adults ages 5-35 years [27].

\section{Anthropometry}

Height was determined using a fixed wall stadiometer, and weight with a digital scale. Height, weight and body mass index (BMI) measurements were expressed as Z-scores. Young adults' Z-scores were calculated assuming a maximum age of 20 years. Pubertal staging was determined by the children or their caregivers with a self-reported Tanner staging questionnaire $[28,29]$.

\section{Statistics}

All results are presented as the median with interquartile range (IQR) as appropriate. SPSS 25 (IBM) and Prism (Graphpad) were used for all statistical analyses. A p-value of $<0.05$ was considered statistically significant, and two-sided testing of the hypothesis was used. 
Spearman rank testing was used for correlations. Kruskal-Wallis ANOVA test for nonnormally distributed data with appropriate corrections for unequal variance, or MannWhitney $\mathrm{U}$ tests were used as required. Bland-Altman analysis was used to plot hip and LS

Page $\mid 8$ DXA measurements and linear regression using the difference between measurements as the dependant variable, and the average of the measurements as the independent variable to analyse the correlation [30]. Fischer's exact test was used to determine the cut-off value at which PTH levels (as a continuous variable expressed in ULN values) best predicted CortBMD below -2SD. All variable with univariate correlations of $p \leq 0.15$ were entered into a stepwise multivariable linear regression analysis where CortBMD was the dependent variable and serum biomarkers and DXA Z-scores were independent variables.

\section{$\underline{\text { Results }}$}

\section{Demographics of study population}

The characteristics of the participants are shown in Table 1.

\section{Patient-level outcomes: bone pain and fractures}

Bone pain was reported by $58 \%$ of patients with a median pain score of 4 (IQR 0 to 5.75). The commonest sites were the lower limbs (38\%), back (25\%), knees (14.7\%) and hips (10.3\%). Most pain was reported to follow activities of daily living such as walking to school or doing housework, and required the use of analgesics. Bone pain score correlated with hip DXA Z-scores $(p=0.04)$ only. It was not associated with a longer dialysis or CKD duration, serum biomarkers or other radiological measurements.

Ten (10\%) participants reported at least one low-trauma fracture (details in Supplemental Table A). Patients who had suffered a previous fracture had a longer dialysis vintage [4.5 (3.6 to 8.2 ) vs 2.1 ( 0.71 to 5.06 ) years respectively, $p=0.04$ ] and longer duration with a low eGFR [10.8 (6.5 to 13.3 ) vs 4.6 (1.9 to 9.6) years respectively, $p=0.008$ ] compared to patients with no fractures. 
Patients who had a history of previous fractures had lower total mineral content [p=0.02, $-2.15(-3.17$ to -0.54$)$ vs $-0.38(-1.46$ to 0.69$)]$ and CSMI [p=0.048, -1.59 (-2.31 to 0.91 ) vs -0.83 ( -1.65 to 0.13$)$ ] compared to patients without a previous fracture; this was despite the fact that the fractures preceded the study imaging by months or years. On multivariable linear regression, DXA and PQCT measures did not associate with a history of past fractures.

\section{Bone Imaging}

Bland-Altman analysis [30] showed that there was very weak agreement between the Z-scores provided by hip and LS DXA $\left[R^{2}=0.06, \beta 0.25(95 \% \mathrm{Cl} 0.05,0.41), p=0.013\right]$. (Figure 1) Both DXA site Z-scores were associated with weight SDS (LS $r=0.23, p<0.05$ and Hip $r=0.49, p<0.0001)$.

Comparing the PQCT BMD Z-scores with the DXA Z-scores, we found that TrabBMD correlated with both hip and LS DXA ( $r=0.66, p<0.0001$ and $r=0.51, p<0.0001)$. CortBMD was not associated with hip or LS DXA Z-scores.

The dialysis group had significantly lower BMD Z-scores by DXA compared to the CKD group, both for LS and hips (Figure 2 \& Supplemental Table B). The dialysis group also had lower cortical and trabecular BMD as well as cortical mineral content compared to the CKD group (Figure 3) as measured by pQCT.

\section{Serum biomarkers}

We examined the correlations of serum biomarkers with imaging modalities (Supplemental Tables $C$ and D). Total calcium showed a positive association both bone compartments separately by pQCT; CortBMD ( $r=0.33, p=0.001$; Figure $4 a)$ and trabBMD $(r=0.32, p=0.001)$. Ionised calcium correlated with cortical measures: CortBMD $(r=0.37$, $p<0.001)$, cortical area $(r=0.21, p=0.04)$ and cortical mineral content $(r=0.26, p=0.01)$. Both PTH and ALP showed inverse correlations with cortical measures: CortBMD ( $r=-0.44$, $p<0.0001$ and $r=-0.22, p<0.03$ respectively) and cortical mineral content $(r=-0.25, p=0.01$ and $r=-0.26, p=0.009$ respectively); Figures $4 b$ and $4 c$. In addition, serum bicarbonate was 
significantly associated with trabBMD $(r=-0.23, p=0.02)$ and hip DXA Z-scores $(r=-0.23$, $\mathrm{p}=0.04)$.

Given that different PTH levels are recommended by KDIGO [9] and the European

Page | 10 Pediatric Dialysis Working Group (EPDWG) [31], we examined the different target PTH levels against CortBMD in our cohort. CortBMD was significantly lower in participants with PTH>9xULN $(-1.92,-3.01$ to -0.64$)$, compared to $2-9 x \operatorname{LLN}(-0.29,-1.56$ to $0.52, p=0.03)$ (Figure 5a). However, there was no difference between PTH levels of $<2 x$ ULN $(-0.12,-0.56$ to 0.69 ) and 2-9xULN ( $p=0.42)$ groups. When applying the EPDWG recommendations, PTH values less than $3 \times$ ULN were associated with significantly higher cortical BMD compared to PTH values $>3 x$ XLN (-0.11 [ -0.56 to 0.79 ], vs -1.35 [-2.98 to 0.01 ], $p<0.0001$ ) (Figure $5 b)$. When PTH values were less than 3xULN none of the patients had a CortBMD below -2SD (OR $95 \% \mathrm{Cl} 7.33$ to infinity) [sensitivity $100 \%$ (95\% $\mathrm{Cl} 93.1 \%$ to $100 \%)$ and specificity $36.9 \%$ (95\% Cl $24.5 \%$ to $51.4 \%)]$. Further analysis confirmed that with increasing PTH levels the odds of having a CortBMD below -2SD increased, such that with PTH $>4 x U L N$, the odds of a CortBMD below -2SD was 11-fold higher [OR 11.08 (95\% $\mathrm{Cl} 3.12$ to 37.90$)$, sensitivity $95 \%$ (95\% Cl $86.3 \%$ to $98.6 \%)$, specificity $36.8 \%(95 \% \mathrm{Cl} 23.4 \%$ to $52.7 \%)$ ].

\section{Medication and Supplements}

More patients in the CKD cohort were on Ca-based phosphate binders compared to the dialysis group (73.1\% vs $36.8 \%$ ). Consequently, the elemental Ca intake from the binders was significantly higher in CKD compared to dialysis patients [p=0.03, 1.06 ( 0 to 2.13 ) vs 0 (0 to 1.46$) \mathrm{mmol} / \mathrm{kg} / \mathrm{day}$. There was no significant difference in alfacalcidol ( $1 \alpha$ hydroxyvitamin D3) treatment ( $p=0.08)$. On univariable analysis alfacalcidol was associated with TrabBMD ( $r=0.27, p=0.02)$, but was no longer significant on multivariable analysis. None of the medications were associated with CortBMD, or DXA Z-scores (Supplemental Table E).

\section{Multivariable regression analysis}


We explored the ability of serum biomarkers and imaging tools used in routine clinical practice to predict CortBMD. We constructed stepwise multivariable linear regression models, including only those parameters with $p \leq 0.15$ on univariable analysis into the models(Table 2). Notably dialysis vintage, number of years with a low eGFR and dialysis/pre-dialysis status were not significant on univariable analysis. Model 1 included all serum biomarkers with $\mathrm{p} \leq 0.15$ on univariable analysis. Model 2 included KDIGO recommended measures ( $\mathrm{Ca}, \mathrm{ALP}$, and $\mathrm{PTH}$ ) for monitoring MBD; phosphate and 25OHD did not reach the pre-defined threshold on univariable analysis. Model 3 replaced total calcium with its co-dependent variable ionised calcium. Model 4 included lumbar spine DXA.

In the most robust model (model 2), independent predictors of CortBMD were total Ca $(\beta$ 0.21, $p=0.005), \operatorname{ALP}(\beta-0.36, p<0.0001)$ and PTH $(\beta-0.43, p<0.0001)$, accounting for $57 \%$ of the variability in CortBMD $\left(R^{2} 0.57\right)$. When all significant variables from univariable analysis were included, bicarbonate was also a significant independent predictor of CortBMD ( $\beta$ 0.16, $p=0.03$; model 1). Including LS DXA did not improve the predictive value of the model (model 4).

\section{Discussion}

Mineral and bone disorders of CKD account for a significant burden of pain and atraumatic fractures even in children and young adults with CKD and on dialysis. Our data show that routinely measured serum biomarkers $\mathrm{Ca}$, PTH and ALP taken together are moderate predictors of BMD, whereas DXA is not a useful measure, and should not be routinely performed in children and young adults with CKD4-5 or on dialysis.

In this cohort of young people under 30 years of age morbidity from CKD-MBD is significant and prevalent. The pain affects daily activities, and requires analgesia. This is consistent with previous studies, showing that clinical symptoms of bone disease are present in children and adolescents on dialysis [32]. In a study reported almost 2 decades ago, young adults with childhood CKD had a heavy burden of bone disease ranging from bone pain to deformities, atraumatic fractures and impaired mobility from bone disease [33]. With improved management, both prevalence and severity of MBD symptoms have reduced over the years, but remain a major concern for our patients [34]. The CKiD study 
(CKD in Children) reported fracture rates of 2.4 and 3 -fold higher in males and females respectively compared to their healthy peers [7]. 16\% reported a prior fracture and over 3.9 years of follow up, $12.5 \%$ had a fracture [7]. $10 \%$ of our cohort had at least one previous low trauma fracture, consistent with the higher fracture risk in the young CKD population. In a study of 89 children with CKD stages 2-5D, 6.5\% suffered a fracture during the one year follow-up, and fracture risk doubled for every 1 SD decrease in CortBMD[14]. We showed that patients with a previous fracture have lower LS BMD. However, the fractures preceded the bone imaging by months or years, and this association should be viewed with some caution. A long-term longitudinal study is required to establish the ability of BMD assessment by DXA or PQCT to predict fractures in the young CKD population.

In our cohort Ca, PTH and ALP, taken together, accounted for only $57 \%$ of variability in CortBMD. Our data are consistent with previous studies showing serum biomarkers are poor surrogate markers in assessing BMD, but as suggested by KDIGO, trends in $\mathrm{Ca}, \mathrm{P}, \mathrm{PTH}$ and ALP, taken together, are the best available measure for clinical management of MBD [2]. Serum biomarkers do not reflect the true state of bone turnover or mineralization [16, 17], and no biomarker individually or in combination can reliably identify mineralization or turnover defects $[18,35]$. Some markers vary significantly with age and stage of growth such as ALP [36]. Studies using bone histology have shown a mineralization defect in over $80 \%$ of the dialysis population; this was associated with higher PTH and lower calcium levels [16, 37]. In fact, mineralization defects can begin early in children, with up to a third in CKD stage 2 having abnormal mineralization [37], even though serum Ca, P and PTH levels may be normal. This is in sharp contrast with bone histology in older adults on dialysis wherein abnormal mineralization is present in $3 \%$ of patients [38]. Other studies report higher ALP levels are associated with higher bone turnover rates by bone biopsy [16], but also lower CortBMD Z-scores by PQCT [22], suggesting an uncoupling of osteoid formation and its mineralization in CKD, as also shown by an inverse correlation of CortBMD and ALP in our cohort.

Alfacalcidol was associated with trabecular BMD on univariable analysis. Previous animal $[39,40]$ and clinical studies $[41,42]$ have shown that $1 \alpha$ hydroxyvitamin D3 reduces bone resorption but maintains and/or stimulates bone formation by increasing osteoblast 
activity. None of the medication or supplements were independent predictors of cortical or trabecular BMD in our cohort.

The assessment of BMD remains challenging and fraught with technical difficulties.

Page | 13 Bone biopsy, although considered the gold standard in assessing mineralization changes, is highly invasive, requires skilled interpretation, and is rarely performed in children or adults with CKD. DXA is widely available, observer independent, and provides aBMD data as wellvalidated age, sex and race matched Z-scores [43]. As a two-dimensional image, DXA superimposes cortical and trabecular bone. PTH has an anabolic effect on trabecular bone and a catabolic effect on cortical bone [44], so effects of hyperparathyroidism cannot be studied with DXA [45]. Trabecular thickness, spacing, connectivity and the ratio of plate- and rod-like structures all contribute to bone strength and quality [46], and DXA provides no information on bone microarchitecture. Given that DXA measures areal BMD $\left(\mathrm{g} / \mathrm{cm}^{2}\right)$ it can underestimate volumetric BMD $\left(\mathrm{g} / \mathrm{cm}^{3}\right)$ in children with short stature [19] and overestimate BMD in a tall child [47], and make serial DXA scanning in growing children particularly challenging. There have been a few studies exploring the use of DXA in the young CKD population, and its association with biochemical markers, but none have examined the association of BMD with fractures [48-50].

Some of the limitations of DXA can be overcome by PQCT which distinguishes between cortical and trabecular bone, and is not affected by patient size. However, pQCT imaging is largely operator dependant, different protocols are used around the world, and the reference data to calculate age, height and sex adjusted Z-scores for healthy people is limited $[27,51,52]$. These drawbacks have limited its use to research. PQCT has been used in the young CKD population to show bone demineralization [22] and correlated a reduction in cortical BMD Z-score with a higher fracture risk in children with CKD 2-5D [14]. It has also shown that cortical BMD is inversely linked to higher PTH and ALP levels [53] as reported in our study.

Our data suggests that serum bicarbonate levels were significantly positively associated with CortBMD. There is evidence that acidosis impairs bone mineralization [54]. Treatment with potassium citrate for 12 months improves cortical parameters on bone biopsy in post-transplant patients $(n=19)$ [55]. In vitro studies show that bone resorption increases with acidosis, through upregulation of osteoclast activity [56] and buffering of the 
acidosis with calcium carbonate released from the bone leads to further demineralization [57]. Thus, it may be important to consider serum bicarbonate alongside routinely measured biomarkers of MBD.

Page | 14 The appropriate target range of PTH levels required for bone mineralization remains heavily debated, and often extrapolated from adult data [9] or historic and highly confounded bone histology data $[18,58-60]$. We examined the predictive value of the KDIGO recommended target range of 2-9xULN (although based on studies in adult CKD cohorts alone) and the significantly lower target of $<3 x U L N$ suggested by the EPDWG. We found that there was a strong non-linear association between PTH and CortBMD such that when PTH was $<3 x U L N$ none of the patients had CortBMD below -2SD. On the other hand, PTH target levels of $<2 x U L N$ and between 2-9xULN were unable to differentiate normal CortBMD, implying a lack of sensitivity of such a wide PTH target. A review of bone biopsy studies in children with CKD suggests that high PTH levels are associated with high bone turnover, but the ability of serum PTH levels to distinguish between low and normal turnover bone disease is less clear [61]. Of note, several bone biopsy studies were either small, in older adults, on patients with long-standing hyperparathyroidism or those who had undergone parathyroidectomy, or patients on aluminium containing phosphate binders, making them less relevant to current practice. In untreated CKD, PTH levels increase early in the course of CKD and skeletal resistance develops requiring even higher PTH levels to maintain normal turnover $[58,61]$.

Registry data [32], prospective cohort studies examining fracture risk [7], longitudinal studies examining cortical BMD using $\mathrm{PQCT}$, growth data as well as vascular imaging studies suggest keeping PTH closer to the normal range. A study from the IPPN registry in over 900 children on peritoneal dialysis found that time-averaged PTH concentrations above $500 \mathrm{pg} / \mathrm{ml}$ were associated with impaired longitudinal growth, PTH levels exceeding $300 \mathrm{pg} / \mathrm{ml}$ increased clinical and radiological symptoms, but the risk of hypercalcemia increased with levels below 100 pg/ml, suggesting a PTH target range of 100$300 \mathrm{pg} / \mathrm{ml}$ in the pediatric age group [32]. Recently, the chronic kidney disease in childhood (CKiD) study has shown a lower fracture risk in children with lower PTH levels [7]. A prospective longitudinal follow-up study in children with CKD2-5D has shown that a greater increase in PTH was associated with greater declines in CortBMD Z-scores over the 1-year 
follow-up period [14]. A concern with a low PTH or suppression below the target range is the development of adynamic bone disease, as reported even in children [62]. Indeed, in the presence of adynamic bone disease, a normal cortical or trabecular BMD does not necessarily imply normal structure and mechanical properties of the bone [63]. However, others have challenged the concept of 'adynamic bone' as children with CKD in whom PTH was kept within normal limits had an above average rate of growth [64], suggesting that normal PTH levels do not cause a low turnover bone state. Importantly, a pediatric dialysis cohort with PTH levels within 2-3xULN had normal carotid intima-media thickness, pulse wave velocity and lower coronary artery calcification scores compared to those with PTH $>3 x$ ULN [65]. Taken together, these data suggest that PTH within 3xULN is associated with CortBMD above -2SD. Longitudinal studies are required to confirm this association.

In this paper we report the initial cross-sectional data from a larger multicentre longitudinal study examining changes in BMD and vascular calcification in children and young adults with CKD4-5D. We continue to gather serial data on serum biomarkers to determine if trends in biomarkers will improve their predictive value in determining BMD. We are also repeating DXA imaging to establish the predictive ability of DXA in identifying patients at risk of fractures. As bone biopsy is highly invasive, we used pQCT as the method of establishing CortBMD. PQCT has remained a research tool due to technical procedural constraints in obtaining the imaging as well as a lack of standardization of the measurement technique [21] and lack of published reference data. We sought to alleviate these by having 2 observers (ADL, NJC) independently score and evaluate the PQCT scans images as per Bleu et al [66].

\section{Conclusion}

In summary, we have shown that CKD-MBD leads to significant morbidity in young patients with a high prevalence of bone pain and atraumatic fractures. Assessment of CortBMD in the absence of bone biopsy is challenging, with routinely measured serum biomarkers $\mathrm{Ca}, \mathrm{PTH}, \mathrm{ALP}$ and bicarbonate being only moderate independent predictors of cortical BMD. DXA imaging does not correlate with CortBMD and should not be routinely 
performed in children and young adults with CKD4-5D. In this young cohort, PTH levels below 3xULN were associated with a normal cortical BMD.

Page | 16

\section{Acknowledgements}

ADL is funded by a joint Kidney Research UK (TF_002_20161124) and Kids Kidney Research Training Fellowship grant (KKR/Paed2017/01).

RS is funded by a National Institute for Health Research (NIHR), (CDF-2016-09-038; Career Development Fellowship) for this research project. This publication presents independent research funded by the National Institute for Health Research (NIHR). The views expressed are those of the author(s) and not necessarily those of the NHS, the NIHR or the Department of Health and Social Care. This work took place in the Biomedical Research Centre at Great Ormond Street Hospital for Children NHS Foundation Trust and University College London.

\section{Conflict of Interest Statements}

All authors have declared no conflict of interest.

The results presented in this paper have not been published previously in whole or part, except in abstract format.

\section{Authors' Contributions}

ADL, RS study conception and design, acquisition of data, analysis and interpretation of data, drafting of manuscript

NJC, MF, CJF interpretation of data, critical revision

JL, MBL provision of Z-scores for PQCT data

VA, AM, LB, AK, MDS, DCW, NDD, JP, DVM, MBL Collaborators at local research sites and Revision of Manuscript 


\section{Funding}

ADL is funded by a joint Kidney Research UK (TF_002_20161124) and a Kids Kidney Research Training Fellowship grant (KKR/Paed2017/01).

Page | 17 MDS acknowledges financial support from the Department of Health via the National Institute for Health Research comprehensive Biomedical Research Centre and Clinical Research Facilities awards to Guy's and St Thomas' NHS Foundation Trust in partnership with King's College London and King's College Hospital NHS Foundation Trust.

RS holds a Career Development Fellowship with the National Institute for Health Research.

\section{References}

1. Wesseling-Perry K, Salusky IB. Phosphate binders, Vitamin D and Calcimimetics in the Management of Chronic Kidney Disease-Mineral Bone Disorders (CKD-MBD) in Children. Pediatric nephrology (Berlin, Germany) 2013;28(4):617-625

2. Ketteler M, Block GA, Evenepoel P, et al. Diagnosis, Evaluation, Prevention, and Treatment of Chronic Kidney Disease-Mineral and Bone Disorder: Synopsis of the Kidney Disease: Improving Global Outcomes 2017 Clinical Practice Guideline Update. Ann Intern Med 2018;168(6):422-430 3. Portale AA, Wolf $\mathrm{M}$, Juppner $\mathrm{H}$, et al. Disordered FGF23 and mineral metabolism in children with CKD. Clin J Am Soc Nephrol 2014;9(2):344-353

4. Portale AA, Wolf MS, Messinger S, et al. Fibroblast Growth Factor 23 and Risk of CKD Progression in Children. Clin J Am Soc Nephrol 2016;11(11):1989-1998

5. Wan M, Smith C, Shah V, et al. Fibroblast growth factor 23 and soluble klotho in children with chronic kidney disease. Nephrol Dial Transplant 2013;28(1):153-161

6. Ketteler M, Block GA, Evenepoel P, et al. Executive summary of the 2017 KDIGO Chronic Kidney\&\#xa0;Disease\&\#x2013;Mineral and Bone Disorder (CKD-MBD) Guideline Update: what\&\#x2019;s changed and why it matters. Kidney International;92(1):26-36

7. Denburg MR, Kumar J, Jemielita T, et al. Fracture Burden and Risk Factors in Childhood CKD: Results from the CKiD Cohort Study. Journal of the American Society of Nephrology : JASN 2016;27(2):543-550

8. Yenchek $\mathrm{RH}, \mathrm{Ix} \mathrm{JH}$, Shlipak MG, et al. Bone mineral density and fracture risk in older individuals with CKD. Clin J Am Soc Nephrol 2012;7(7):1130-1136

9. Ketteler M, Block GA, Evenepoel P, et al. Executive summary of the 2017 KDIGO Chronic Kidney Disease-Mineral and Bone Disorder (CKD-MBD) Guideline Update: what's changed and why it matters. Kidney International;92(1):26-36

10. limori S, Mori Y, Akita W, et al. Diagnostic usefulness of bone mineral density and biochemical markers of bone turnover in predicting fracture in CKD stage 5D patients--a singlecenter cohort study. Nephrol Dial Transplant 2012;27(1):345-351

11. Naylor KL, Garg AX, Zou G, et al. Comparison of fracture risk prediction among individuals with reduced and normal kidney function. Clin J Am Soc Nephrol 2015;10(4):646-653

12. West SL, Lok CE, Langsetmo $\mathrm{L}$, et al. Bone mineral density predicts fractures in chronic kidney disease. J Bone Miner Res 2015;30(5):913-919 
13. Weaver CM, Gordon CM, Janz KF, et al. The National Osteoporosis Foundation's position statement on peak bone mass development and lifestyle factors: a systematic review and implementation recommendations. Osteoporos Int 2016;27(4):1281-1386

14. Denburg MR, Tsampalieros AK, de Boer IH, et al. Mineral Metabolism and Cortical Volumetric Bone Mineral Density in Childhood Chronic Kidney Disease. The Journal of Clinical Endocrinology and Metabolism 2013;98(5):1930-1938

15. Ott SM. Histomorphometric measurements of bone turnover, mineralization, and volume. Clin J Am Soc Nephrol 2008;3 Suppl 3:S151-156

16. Bakkaloglu SA, Wesseling-Perry K, Pereira RC, et al. Value of the new bone classification system in pediatric renal osteodystrophy. Clin J Am Soc Nephrol 2010;5(10):1860-1866

17. Barreto FC, Barreto DV, Moysés RMA, et al. K/DOQI-recommended intact PTH levels do not prevent low-turnover bone disease in hemodialysis patients. Kidney International 2008;73(6):771777

18. Sprague SM, Bellorin-Font E, Jorgetti V, et al. Diagnostic Accuracy of Bone Turnover Markers and Bone Histology in Patients With CKD Treated by Dialysis. Am J Kidney Dis 2016;67(4):559-566

19. Zemel BS, Leonard MB, Kelly $\mathrm{A}$, et al. Height adjustment in assessing dual energy $\mathrm{x}$-ray absorptiometry measurements of bone mass and density in children. J Clin Endocrinol Metab 2010;95(3):1265-1273

20. Haffner D, Schaefer F, Nissel R, et al. Effect of growth hormone treatment on the adult height of children with chronic renal failure. German Study Group for Growth Hormone Treatment in Chronic Renal Failure. N Engl J Med 2000;343(13):923-930

21. Lalayiannis AD, Crabtree NJ, Fewtrell $M$, et al. Assessing bone mineralisation in children with chronic kidney disease: what clinical and research tools are available? Pediatr Nephrol 2019

22. Wetzsteon RJ, Kalkwarf HJ, Shults J, et al. Volumetric Bone Mineral Density and Bone Structure in Childhood Chronic Kidney Disease. Journal of Bone and Mineral Research 2011;26(9):2235-2244

23. Schwartz GJ, Munoz A, Schneider MF, et al. New equations to estimate GFR in children with CKD. J Am Soc Nephrol 2009;20(3):629-637

24. Jensen MP, Turner JA, Romano JM. What is the maximum number of levels needed in pain intensity measurement? Pain 1994;58(3):387-392

25. Crabtree NJ, Arabi A, Bachrach LK, et al. Dual-energy X-ray absorptiometry interpretation and reporting in children and adolescents: the revised 2013 ISCD Pediatric Official Positions. J Clin Densitom 2014;17(2):225-242

26. Crabtree NJ, Shaw NJ, Bishop NJ, et al. Amalgamated Reference Data for Size-Adjusted Bone Densitometry Measurements in 3598 Children and Young Adults-the ALPHABET Study. J Bone Miner Res 2017;32(1):172-180

27. Leonard MB, Elmi A, Mostoufi-Moab S, et al. Effects of Sex, Race, and Puberty on Cortical Bone and the Functional Muscle Bone Unit in Children, Adolescents, and Young Adults. The Journal of Clinical Endocrinology and Metabolism 2010;95(4):1681-1689

28. Marshall WA, Tanner JM. Variations in pattern of pubertal changes in girls. Arch Dis Child 1969;44(235):291-303

29. Marshall WA, Tanner JM. Variations in the pattern of pubertal changes in boys. Arch Dis Child 1970;45(239):13-23

30. Bland JM, Altman DG. Statistical methods for assessing agreement between two methods of clinical measurement. Lancet 1986;1(8476):307-310

31. Klaus G, Watson A, Edefonti A, et al. Prevention and treatment of renal osteodystrophy in children on chronic renal failure: European guidelines. Pediatr Nephrol 2006;21(2):151-159

32. Borzych D, Rees L, Ha IS, et al. The bone and mineral disorder of children undergoing chronic peritoneal dialysis. Kidney Int 2010;78(12):1295-1304

33. Groothoff JW, Offringa M, Van Eck-Smit BL, et al. Severe bone disease and low bone mineral density after juvenile renal failure. Kidney Int 2003;63(1):266-275 
34. Tong A, Manns B, Wang AYM, et al. Implementing core outcomes in kidney disease: report of the Standardized Outcomes in Nephrology (SONG) implementation workshop. Kidney international 2018;94(6):1053-1068

35. Soeiro EMD, Castro L, Menezes R, et al. Association of parathormone and alkaline phosphatase with bone turnover and mineralization in children with CKD on dialysis: effect of age, gender, and race. Pediatr Nephrol 2020

36. Zierk J, Arzideh F, Haeckel R, et al. Pediatric reference intervals for alkaline phosphatase. Clin Chem Lab Med 2017;55(1):102-110

37. Wesseling-Perry K, Pereira RC, Tseng $\mathrm{CH}$, et al. Early skeletal and biochemical alterations in pediatric chronic kidney disease. Clin J Am Soc Nephrol 2012;7(1):146-152

38. Malluche HH, Mawad HW, Monier-Faugere MC. Renal osteodystrophy in the first decade of the new millennium: analysis of 630 bone biopsies in black and white patients. J Bone Miner Res 2011;26(6):1368-1376

39. Chen H, Tian X, Liu X, et al. Alfacalcidol-stimulated focal bone formation on the cancellous surface and increased bone formation on the periosteal surface of the lumbar vertebrae of adult female rats. Calcif Tissue Int 2008;82(2):127-136

40. Shiraishi A, Takeda S, Masaki T, et al. Alfacalcidol Inhibits Bone Resorption and Stimulates Formation in an Ovariectomized Rat Model of Osteoporosis: Distinct Actions from Estrogen. Journal of Bone and Mineral Research 2000;15(4):770-779

41. Felsenberg $\mathrm{D}$, Bock $\mathrm{O}$, Borst $\mathrm{H}$, et al. Additive impact of alfacalcidol on bone mineral density and bone strength in alendronate treated postmenopausal women with reduced bone mass. J Musculoskelet Neuronal Interact 2011;11(1):34-45

42. El-Agroudy AE, El-Husseini AA, El-Sayed M, et al. A prospective randomized study for prevention of postrenal transplantation bone loss. Kidney Int 2005;67(5):2039-2045

43. Gordon CM, Leonard MB, Zemel BS. 2013 Pediatric Position Development Conference: executive summary and reflections. J Clin Densitom 2014;17(2):219-224

44. Parfitt AM. A structural approach to renal bone disease. J Bone Miner Res 1998;13(8):12131220

45. Weber LT, Mehls $\mathrm{O}$. Limitations of dual $\mathrm{x}$-ray absorptiometry in children with chronic kidney disease. Pediatr Nephrol 2010;25(1):3-5

46. Mittra E, Rubin C, Qin YX. Interrelationship of trabecular mechanical and microstructural properties in sheep trabecular bone. J Biomech 2005;38(6):1229-1237

47. Crabtree N, Ward K. Bone Densitometry: Current Status and Future Perspective. Endocr Dev 2015;28:72-83

48. Pluskiewicz W, Adamczyk P, Drozdzowska B, et al. Skeletal status in children and adolescents with chronic renal failure before onset of dialysis or on dialysis. Osteoporos Int 2003;14(4):283-288

49. Waller S, Ridout $D$, Rees $L$. Bone mineral density in children with chronic renal failure. Pediatr Nephrol 2007;22(1):121-127

50. Griffin LM, Kalkwarf HJ, Zemel BS, et al. Assessment of dual-energy X-ray absorptiometry measures of bone health in pediatric chronic kidney disease. Pediatr Nephrol 2012;27(7):1139-1148

51. Adams JE, Engelke K, Zemel BS, et al. Quantitative computer tomography in children and adolescents: the 2013 ISCD Pediatric Official Positions. J Clin Densitom 2014;17(2):258-274

52. Roggen I, Roelants $M$, Sioen I, et al. Pediatric reference values for tibial trabecular bone mineral density and bone geometry parameters using peripheral quantitative computed tomography. Calcif Tissue Int 2015;96(6):527-533

53. Lima EM, Goodman WG, Kuizon BD, et al. Bone density measurements in pediatric patients with renal osteodystrophy. Pediatr Nephrol 2003;18(6):554-559

54. Raphael KL. Metabolic Acidosis and Subclinical Metabolic Acidosis in CKD. Journal of the American Society of Nephrology : JASN 2018;29(2):376-382 
55. Starke A, Corsenca A, Kohler T, et al. Correction of metabolic acidosis with potassium citrate in renal transplant patients and its effect on bone quality. Clin J Am Soc Nephrol 2012;7(9):1461-

1472

56. Yuan F-L, Xu M-H, Li X, et al. The Roles of Acidosis in Osteoclast Biology. Frontiers in physiology 2016;7:222-222

Page 120 57. K/DOQI clinical practice guidelines for bone metabolism and disease in chronic kidney disease. Am J Kidney Dis 2003;42(4 Suppl 3):S1-201

58. Ballanti $P$, Wedard BM, Bonucci E. Frequency of adynamic bone disease and aluminum storage in Italian uraemic patients--retrospective analysis of 1429 iliac crest biopsies. Nephrol Dial Transplant 1996;11(4):663-667

59. Salusky IB, Ramirez JA, Oppenheim W, et al. Biochemical markers of renal osteodystrophy in pediatric patients undergoing CAPD/CCPD. Kidney Int 1994;45(1):253-258

60. Coen G, Ballanti P, Bonucci E, et al. Bone markers in the diagnosis of low turnover osteodystrophy in haemodialysis patients. Nephrol Dial Transplant 1998;13(9):2294-2302

61. Rees $\mathrm{L}$. What parathyroid hormone levels should we aim for in children with stage 5 chronic kidney disease; what is the evidence? Pediatric nephrology (Berlin, Germany) 2008;23(2):179-184

62. Ziolkowska H, Paniczyk-Tomaszewska M, Debinski A, et al. Bone biopsy results and serum bone turnover parameters in uremic children. Acta Paediatr 2000;89(6):666-671

63. $\mathrm{Ng} \mathrm{AH}$, Omelon S, Variola F, et al. Adynamic Bone Decreases Bone Toughness During Aging by Affecting Mineral and Matrix. J Bone Miner Res 2016;31(2):369-379

64. Waller $\mathrm{S}$, Ledermann S, Trompeter $\mathrm{R}$, et al. Catch-up growth with normal parathyroid hormone levels in chronic renal failure. Pediatr Nephrol 2003;18(12):1236-1241

65. Shroff RC, Donald AE, Hiorns MP, et al. Mineral metabolism and vascular damage in children on dialysis. J Am Soc Nephrol 2007;18(11):2996-3003

66. Blew RM, Lee VR, Farr JN, et al. Standardizing evaluation of pQCT image quality in the presence of subject movement: qualitative versus quantitative assessment. Calcified tissue international 2014;94(2):202-211

Tables

Table 1. Participant descriptive data

\begin{tabular}{|l|l|l|l|}
\hline & CKD participants & Dialysis participants & $\begin{array}{l}\text { Between } \\
\text { group } \\
\text { comparison }\end{array}$ \\
\hline Total, $\mathrm{n}=(\%)$ & $\mathrm{n}=26(25 \%)$ & $\mathrm{n}=77(75 \%)$ & \\
\hline Ages, years median (IQR $\left.{ }^{1}\right)$ & $11.9(6.9-13.8)$ & $14.2(10.9-18.2)$ & $\mathrm{p}=0.008$ \\
\hline $\begin{array}{l}5-19 \text { years, } \mathrm{n}=(\%) \\
20-30 \text { years, } \mathrm{n}=(\%)\end{array}$ & $\begin{array}{l}26(100 \%) \\
0(0 \%)\end{array}$ & $\begin{array}{l}59(76.6 \%) \\
18(23.4 \%)\end{array}$ & $\mathrm{NS}$ \\
\hline $\begin{array}{l}\text { Sex, Female } \mathrm{n}=(\%) \\
18(69.2 \%)\end{array}$ & $38(49.4 \%)$ & $\mathrm{N} / \mathrm{A}$ \\
\hline $\begin{array}{l}\text { Race, } \mathrm{n}= \\
\text { Caucasian/ Asian/ Black/ Other }\end{array}$ & $20 / 2 / 4 / 0$ & $35 / 24 / 17 / 1$ & $\mathrm{NS}=0.01$ \\
\hline
\end{tabular}




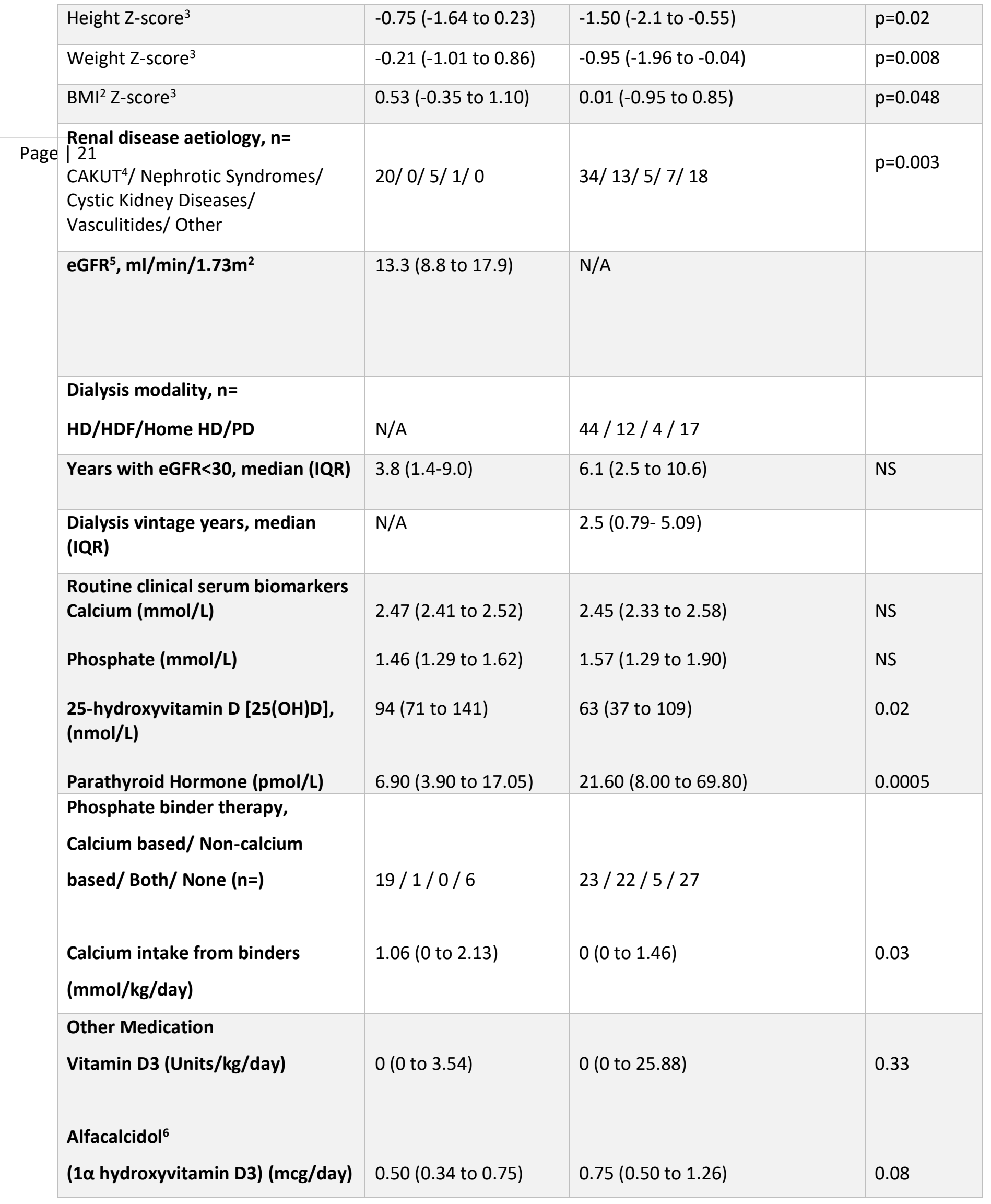


${ }^{1}$ Interquartile range, ${ }^{2}$ Body Mass Index $\left(\mathrm{kg} / \mathrm{m}^{2}\right)$, ${ }^{3}$ Height/Weight/BMI Z-scores calculated for participants aged 5-20 years. Any participants $>20$ years old, Z-scores calculated assuming maximum age of 20 years, ${ }^{4}$ Congenital abnormalities of the kidneys and urinary tract, ${ }^{5}$ estimated Glomerular filtration rate, ${ }^{6}$ Most participants were on alfacalcidol. Three participants in the dialysis group were on paricalcitol and were not included in the medication analysis, NS Not Significant

Table 2. Multivariable linear regression models

\begin{tabular}{|c|c|c|c|c|c|c|c|c|}
\hline $\begin{array}{l}\text { Regression } \\
\text { Model }\end{array}$ & & B & $\begin{array}{c}95.0 \% \\
\text { Confidence } \\
\text { Interval for B }\end{array}$ & $\begin{array}{l}\text { Std. } \\
\text { Error }\end{array}$ & $\begin{array}{c}\text { Standardized } \\
\beta\end{array}$ & p-value & $\begin{array}{l}\text { Model } \\
\mathbf{R}^{2}\end{array}$ & $\begin{array}{l}\text { Model } \\
\text { Adjusted } \\
\mathbf{R}^{\mathbf{2}}\end{array}$ \\
\hline \multirow[t]{6}{*}{1} & (Constant) & -7.75 & $-12.67,-2.82$ & 2.48 & & 0.002 & 0.53 & 0.51 \\
\hline & Total Ca & 2.55 & $0.76,4.35$ & 0.90 & 0.22 & 0.006 & & \\
\hline & $\mathrm{Mg}$ & -0.14 & $-2.04,1.76$ & 0.96 & -0.01 & 0.884 & & \\
\hline & ALP & -0.004 & $-0.01,-0.002$ & 0.001 & -0.37 & $<0.0001$ & & \\
\hline & PTH & -0.015 & $-0.02,-0.01$ & 0.003 & -0.38 & $<0.0001$ & & \\
\hline & Bicarbonate & 0.09 & $0.01,0.17$ & 0.04 & 0.16 & 0.03 & & \\
\hline \multirow[t]{4}{*}{2} & (Constant) & -5.66 & $-10.11,-1.20$ & 2.25 & & 0.01 & 0.58 & 0.57 \\
\hline & Total Ca & 2.57 & $0.79,4.35$ & 0.90 & 0.21 & 0.005 & & \\
\hline & ALP & -0.004 & $-0.01,-0.002$ & 0.001 & -0.36 & $<0.0001$ & & \\
\hline & PTH & -0.02 & $-0.21,-0.10$ & 0.003 & -0.43 & $<0.0001$ & & \\
\hline \multirow[t]{4}{*}{3} & (Constant) & 0.58 & $-0.89,2.04$ & 0.74 & & 0.44 & 0.57 & 0.56 \\
\hline & Ionized Ca & 0.15 & $-1.03,1.32$ & 0.59 & 0.02 & 0.81 & & \\
\hline & ALP & -0.003 & $-0.01,-0.002$ & 0.001 & -0.34 & $<0.0001$ & & \\
\hline & PTH & -0.02 & $-0.02,-0.01$ & 0.003 & -0.53 & $<0.0001$ & & \\
\hline \multirow[t]{5}{*}{4} & (Constant) & -5.25 & $-9.68,-0.81$ & 2.23 & & 0.02 & 0.59 & 0.58 \\
\hline & Total Ca & 2.36 & $0.59,4.13$ & 0.89 & 0.19 & 0.01 & & \\
\hline & ALP & -0.003 & $-0.005,-0.002$ & 0.001 & -0.34 & $<0.0001$ & & \\
\hline & PTH & -0.02 & $-0.02,-0.01$ & 0.003 & -0.42 & $<0.0001$ & & \\
\hline & $\begin{array}{l}\text { Lumbar } \\
\text { spine DXA }\end{array}$ & 0.14 & $-0.06,0.34$ & 0.10 & 0.09 & 0.18 & & \\
\hline
\end{tabular}

Stepwise multivariable linear regression models used to find the best serum biomarkers and/or DXA imaging to predict cortical BMD. All variable with univariate correlations of $p \leq 0.15$ were entered into the analysis. Model 1 included all serum biomarkers meeting the univariate correlation threshold. Model 2 included KDIGO recommended measures ( $\mathrm{Ca}$, ALP, and PTH) for monitoring MBD. Model 3 replaced total calcium with its co-dependent variable ionised calcium. Model 4 included lumbar spine DXA. 


\section{Legends to Figures}

Figure 1. Bland-Altman plot of the mean and difference of agreement between hip and lumbar spine DXA Z-score for the CKD and dialysis participants. +/- 1 standard deviation (SD) lines shown to Page | 23 illustrate the significant difference clinically between the two measures

Figure 2. Violin plot of the lumbar spine and hip Z-scores of the CKD and dialysis groups of participants. Lines show the median and the interquartile range. $p$-value between group significance shown.

Figure 3. Violin plot of the cortical bone mineral density (CortBMD), trabecular BMD (TrabBMD), and cortical mineral content (CortCNT) as measured by PQCT in the CKD and dialysis groups. Lines show the median and the interquartile range. p-value between group significance shown.

Figures $4 a \& 4 b$. Plot of cortical bone mineral density Z-scores according to PTH, total calcium (Ca) and alkaline phosphatase (ALP) values. PTH plotted on a logarithmic scale- log2 (pmol/L). Solid line is linear regression best fit for all participants, with dotted lines showing the $95 \% \mathrm{Cl}$. The $\mathrm{Y}$ axis of CortBMD Z-scores has been curtailed at -6 for consistency. This leaves 3 value points outside the plotted areas as the participants had CortBMD Z-scores lower than -6 . These 3 dialysis participants although outliers, had consistently lower bone imaging Z-scores across all modalities. [Multivariable regression associations for CKD participants only: $R^{2} 0.24$, Ca $\beta=0.12, p=0.55 ; A L P \beta=-0.38, p=0.06$; PTH $\beta=-0.32, p=0.14$; For dialysis participants only: $R^{2} 0.58$, Ca $\beta=0.22, p=0.01 ; A L P \beta=-0.37$, $p<0.0001 ;$ PTH $\beta=-0.41, p<0.0001]$

Figures $5 a, 5 b, 5 c$. Scatter plots of the cortical BMD Z-scores, separated into columns according to the PTH upper limit of normal (ULN) target in different guidelines. Lines show the median and the interquartile range_for all participants. p-value between group significance shown.

\section{Figures}

Figure 1 
Page | 24

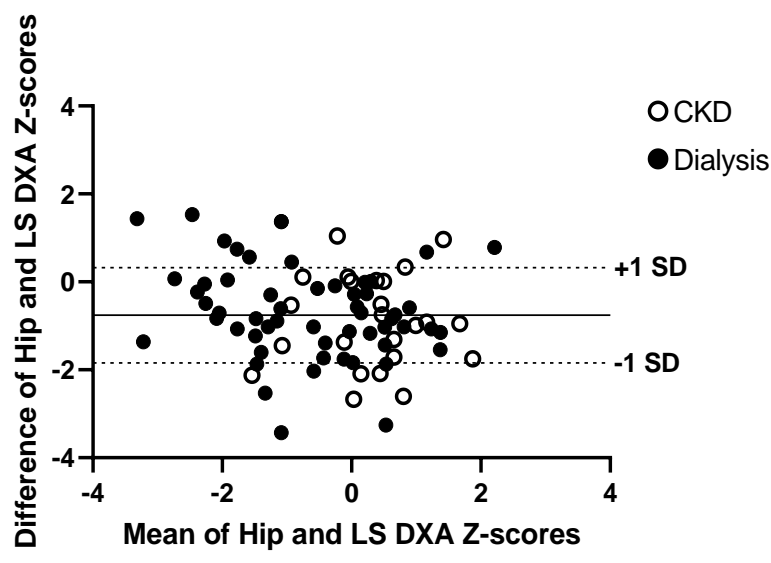

Figure 2

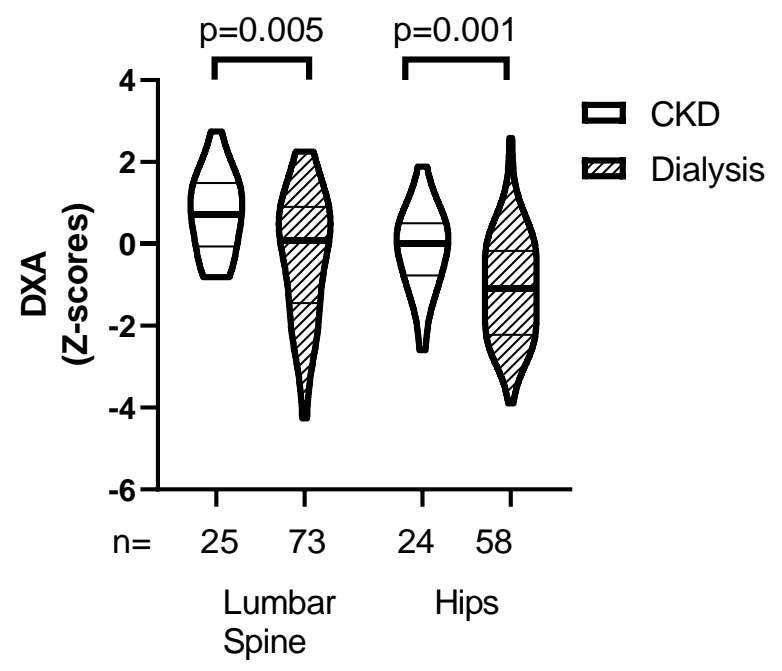


Figure 3

Page | 25

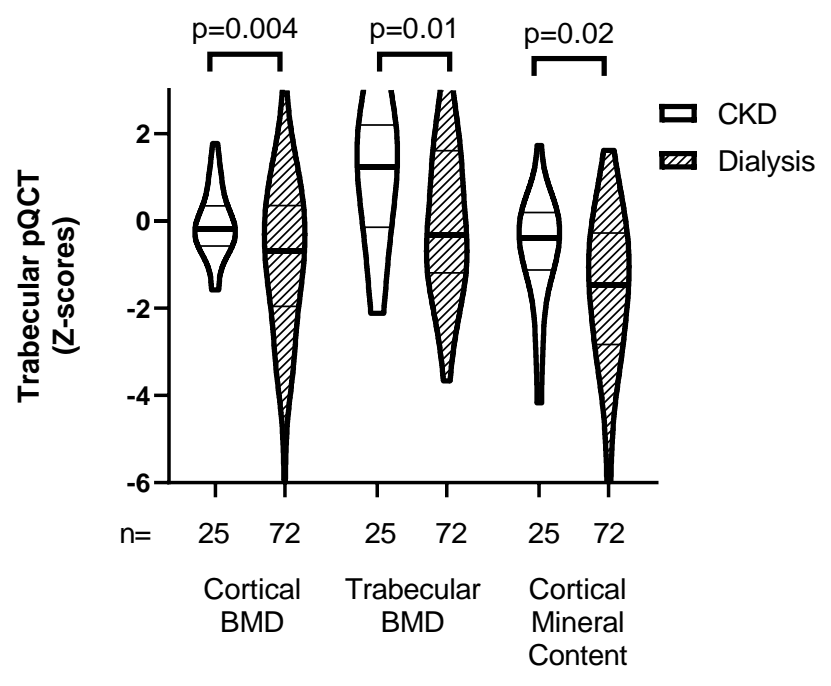

Figures $4 a, 4 b \& 4 c$
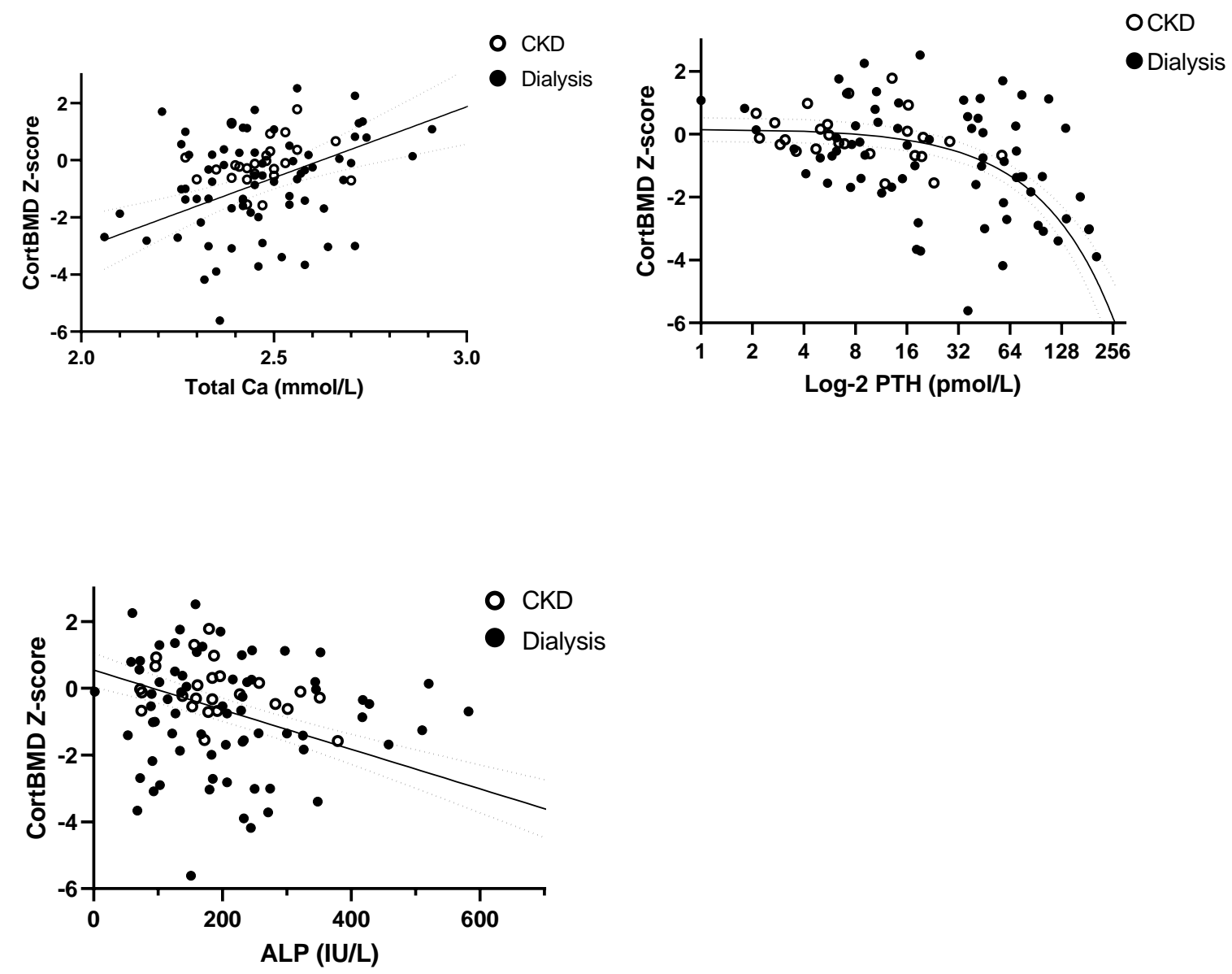
Figures $5 a \& 5 b$

Page | 26
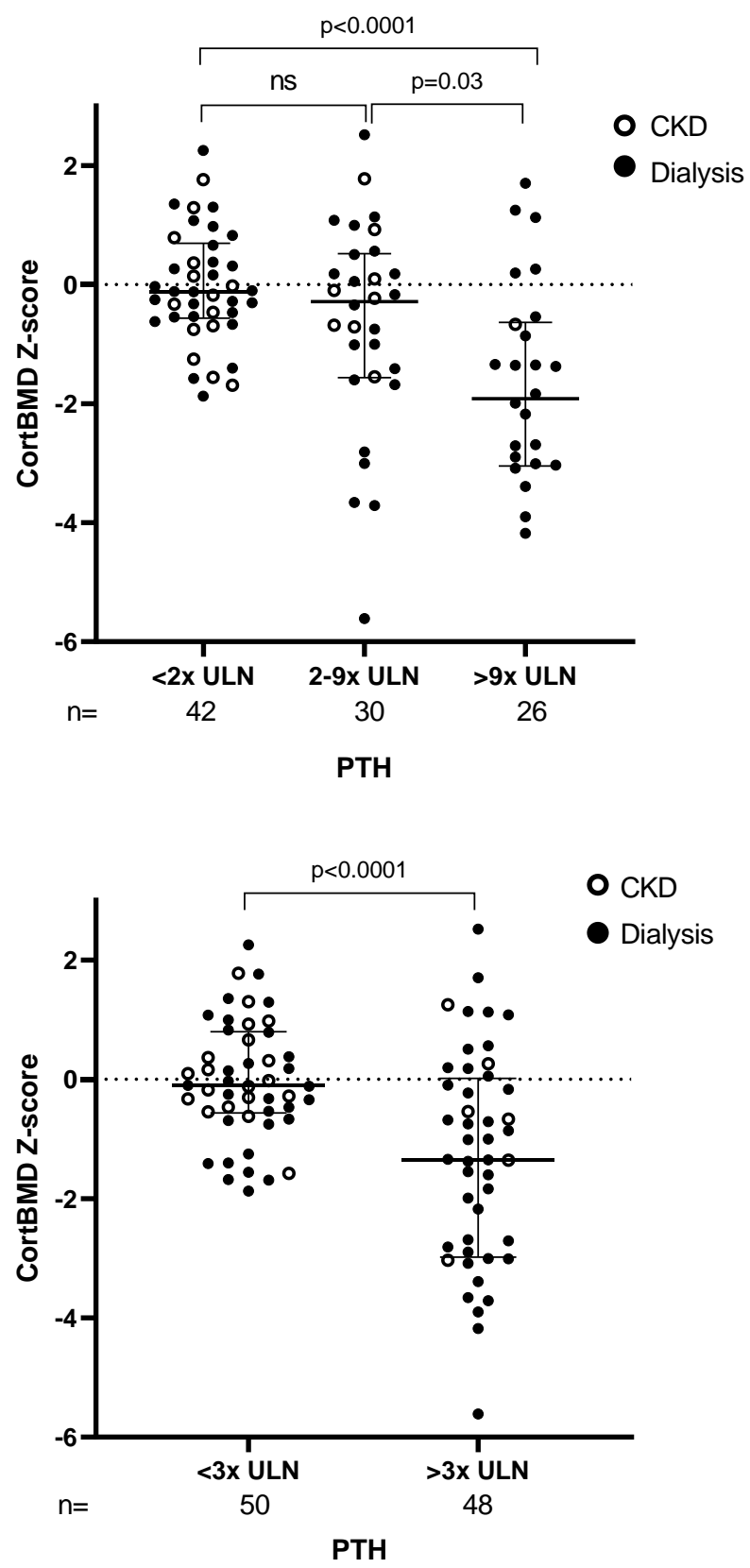
Page | 27 